\title{
BIBLIOGRAPHY
}

1. S. Saks, Theory of the integral, trans. by L. C. Young, Warsaw, 1937, p. 117.

2. F. Hausdorff, Mengenlehre, New York, 1944, p. 253.

UNIVERSITY OF OKLAHOMA

\section{NOTE ON ZEROS OF THE HERMITE POLYNOMIALS AND WEIGHTS FOR GAUSS' MECHANICAL QUADRATURE FORMULA}

\section{J. BARKLEY ROSSER}

The main purpose of this note is to point out that, in most cases, use of the mechanical quadrature formula to compute integrals of the form

$$
\int_{-\infty}^{\infty} e^{-x^{2}} f(x) d x
$$

is unsatisfactory. One can easily see why this would be the case. In a closed interval one can approximate very closely to a continuous function by a high degree polynomial. However, outside the interval, the high degree polynomial will in most cases diverge very markedly from the given function. In (1), this divergence is eventually brought under control by the exponential, but often not in time to prevent a sizeable error.

We illustrate with a numerical example using coefficients from [1],1 and from the end of section 22 of [2]. It can be shown that

$$
\begin{aligned}
\int_{-\infty}^{\infty} \frac{e^{-y^{2}}}{1+y^{2}} d y & =2 \pi^{1 / 2} e\left\{\frac{\pi^{1 / 2}}{2}-\int_{0}^{1} e^{-y^{2}} d y\right\} \\
& =1.34329 \ldots
\end{aligned}
$$

(see [2, Theorem 1-2 and formula (1-17)]). However the quadrature formulas for $n=2,10$, and 16 give

$$
\begin{array}{ll}
n=2, & 1.18164, \\
n=10, & 1.34164, \\
n=16, & 1.34313 .
\end{array}
$$

Received by the editors January 3, 1949 and, in revised form, February 9, 1949.

1 Numbers in brackets refer to the bibliography at the end of the paper. 
Thus, even for $n=16$, we have only a fair approximation, and the computation for $n=16$ is fairly laborious.

In [1], the quadrature formula was applied to

$$
\int_{-\infty}^{\infty} e^{-x^{2}} \cos x d x
$$

with considerable success. However, this is a very special case, since for this integral the asymptotic series actually converges, and rapidly enough that computation by the asymptotic series would be less laborious than using the quadrature formula. The asymptotic series for this integral is obtained by expanding $\cos x$ in the familiar Maclaurin series and integrating term by term.

It is probably true in most cases that if $f(x)$ is any decently differentiable function, then there will be less laborious means of computing (1) than numerical quadrature, so that numerical quadrature should be reserved for the case where $f(x)$ is known only through its values at some points. Further, one should not expect any great accuracy from the numerical quadrature in the majority of cases.

\section{BiBLIOGRAPHY}

1. Robert E. Greenwood and J. J. Miller, Zeros of the hermite polynomials and weights for Gauss' mechanical quadrature formula, Bull. Amer. Math. Soc. vol. 54 (1948) pp. 765-769.

2. J. Barkley Rosser, Theory and application of $\int_{0}^{a} e^{-x^{2}} d x$ and $\int_{0}^{a} e^{-p^{2} v^{2}} d y \int_{0}^{2} e^{-x^{2}} d x$, Part I. Methods of computation, Mapleton House, 5415 Seventeenth Ave., Brooklyn 4, N. Y., 1948. An earlier edition of this was published by the government in 1945 and distributed to 120 libraries around the country, and so is reasonably available.

CORNELl University 